\title{
EDITORIAL
}

\section{¿LA PREVENCIÓN Y EL CONTROL DE LA TOXOPLASMOSIS SON METAS DIFÍCILES DE LOGRAR?}

Un estudio reciente realizado en donantes de sangre del Hemocentro del Café en la ciudad de Manizales, en el que se buscaba la presencia de ADN de Toxoplasma, así como también la presencia de anticuerpos frente a este parásito, mostró que la frecuencia de anticuerpos fue del $34 \%$. Este valor se acerca al promedio nacional descrito en 1988 y además es similar a lo encontrado en otros estudios hechos en Manizales.

Este hallazgo indica que, a pesar de que esta cifra es inferior a las obtenidas en otros estudios, sigue siendo muy similar y además es alta indicando que una de cada tres personas está infectada con el parásito. También, se ha demostrado que la probabilidad de infección aumenta con la edad, pues las personas jóvenes presentan baja frecuencia de anticuerpos y a medida que la edad aumenta la frecuencia de anticuerpos también, llegándose a un pico del $50 \%$ en personas entre los 46 y 55 años de edad. Siendo este grupo de edad el que presenta mayor riesgo a la infección.

La toxoplasmosis, a pesar de las implicaciones clínicas que genera, se considera como una enfermedad de poca importancia a nivel epidemiológico. Sin embargo, la Organización Mundial de la Salud la incluye dentro de las enfermedades infecciosas desatendidas que tiene importancia epidemiológica. Es grave cuando ataca a personas inmunosuprimidas, pero es especialmente preocupante en mujeres embarazadas que se primoinfectan por las posibles secuelas en el feto o recién nacido por la transmisión vertical del parásito. La enfermedad puede afectar ente 3,4 por 1.000 a 6 por 10.000 nacidos vivos dependiendo de la prevalencia (1). También, hay algunos reportes que han asociado la infección crónica con entidades clínicas como la esquizofrenia, posiblemente debido a la predilección que tiene el parásito por el sistema nervioso central (2), cuya invasión puede generar lesiones neuronales que podrían estar asociadas con dicha enfermedad siquiátrica. Este hallazgo podría ser un factor importante para determinar si una persona seropositiva, a pesar de ser inmunocompetente, debe ser tratada o no.

La prevención de la infección es complicada ya que las formas infectantes, y en especial los ooquistes que surgen del proceso infeccioso ocurrido en felinos, pueden contaminar diferentes alimentos. El agua se ha considerado como una buena fuente del parásito. Las verduras crudas y también las carnes de otros animales domésticos como cerdos, bovinos y aves pueden ser un factor de riesgo para la adquisición de la infección cuando se consumen crudas o mal cocidas. Debido a la amplia variedad de fuentes de infección la probabilidad de adquisición del parásito aumenta.

La posible abundancia de gatos ferales en nuestro departamento es una de las causas más probables de los altos índices de infección en la población. Estos felinos al vivir libres deben alimentarse de otros animales como pájaros, roedores y reptiles adquiriendo el parásito, el cual hace su ciclo enteroepitelial y libera millones de ooquistes que van a contaminar la tierra y posteriormente infectar la amplia variedad de hospederos intermediarios y paraténicos como el hombre, que van a contribuir a perpetuar el ciclo de vida del parásito. Además, sus costumbres de deambular por el peridomicilio contribuyen a aumentar la probabilidad de infección de las personas ya que los vectores mecánicos, tales como moscas y en especial las cucarachas, podrían contribuir a la contaminación de alimentos al transportar las formas infectantes del parásito desde las heces del gato a los alimentos dejados al aire libre en las cocinas de las casas. Otro factor que sirve de evidencia a este mecanismo de adquisición de la toxoplasmosis es el hecho de que la frecuencia de infección es mayor en personas que viven en zonas rurales que en urbanas, ya que es más probable la presencia de gatos y también la presencia de vectores mecánicos. 
A partir de lo anterior, se podría inferir que una buena medida para controlar la alta frecuencia de toxoplasmosis en nuestra región sería disminuir la población de gatos silvestres. Para ello, se requeriría implementar medidas como la esterilización tanto de machos y de hembras que disminuyan la población felina silvestre. Esta medida no tendría un efecto positivo a corto plazo ya que la abundancia de ooquistes en el ambiente podría proveer una buena fuente de formas infectantes por varios años, pero la reducción de la población felina podría también disminuir la cantidad de ooquistes en el medio ambiente y de esta manera la probabilidad de adquisición de la infección. Evidencias de que esta medida podría tener éxito se han demostrado en un estudio, en el cual se encontró que la seroconversión es más rápida en cerdos criados en sitios con alta densidad de gatos que en aquellos criados donde la densidad es baja (3). Dicho hallazgo refuerza la importancia de la cantidad de ooquistes en el ambiente frente a la probabilidad de infección de una mayor cantidad de personas.

Esta medida podría ser fuertemente controvertida por los ecologistas, quizás por el desbalance que se produciría en la naturaleza, ya que los felinos por su naturaleza predadora sirven para controlar el número de animales que le sirven de alimento. Sin embargo, el beneficio desde el punto de vista de la salud humana podría ser significativo frente al desbalance. Esta medida, por lo tanto, debería ir acompañada de estudios que demuestren cuál es el impacto negativo de la disminución de la población felina en el ecosistema.

La otra alternativa es la educación en la prevención de la enfermedad, que debería implementarse desde etapas muy tempranas del proceso educativo, que permita concientizar a las personas de la importancia de estas medidas no solo para evitar la toxoplasmosis sino también otras enfermedades infecciosas. El consumo de carnes bien cocidas, de agua potable y ojalá hervida, el correcto manejo de verduras y sobre todo la adecuada manutención de los felinos en cuanto a la alimentación con concentrados y evitar su salida al exterior de las casas sin supervisión, serían factores importantes para controlar y disminuir la frecuencia de la toxoplasmosis en el hombre. Esta estrategia podría ser igualmente efectiva, pero se requeriría de la concientización del Ministerio de Salud Pública de Colombia junto con el Ministerio de Educación en la importancia de incluir dentro del recorrido curricular de las asignaturas correspondientes, tanto en la básica primaria como en la educación media y aun en la educación superior, los contenidos que orienten hacia aspectos básicos de prevención de las enfermedades infecciosas.

JORGE ENRIQUE PÉREZ CÁRDENAS

Director Revista Biosalud

Facultad de Ciencias para la Salud

Universidad de Caldas

\section{REFERENCIAS}

1. Torgerson PR, Mastroiacov P. The global burden of congenital toxoplasmosis: a systematic review. Bull World Health Organ 2013; 91:501-508.

2. Torrey EF, Bartko JJ, Yolken RH. Toxoplasma gondii and Other Risk Factors for Schizophrenia: An Update. Schizophrenia Bulletin 2012; 38:642-64.

3. Ortega-Pacheco A, Acosta-Viana KY, Guzman-Marin E, Uitzil-Álvarez B, Rodríguez-Buenfil JC, JiménezCoello M. Infection dynamic of Toxoplasma gondii in two fattening pig farms exposed to high and low cat density in an endemic region. Veterinary Parasitology 2011; 175:367-371. 